\section{Fehler bei der Medikamentenverordnung im perioperativen HNO-Bereich}

Fehler bei der perioperativen Arzneimittelverordnung und -einnahme speziell im HNO-Bereich sind bisher kaum untersucht worden. In einer umfangreichen prospektiven Beobachtungsstudie wurden Daten aus dem ambulanten und stationären Sektor gesammelt.

E ehler bei der perioperativen Arzneimittelverordnung und -einnahme speziell im HNO-Bereich sind bisher kaum untersucht worden. In einer umfangreichen prospektiven Beobachtungsstudie wurden Daten aus dem ambulanten und stationären Sektor gesammelt. Durchgeführt wurde die deskriptive Studie an einem großen HNO-Zentrum der Universität Florida in Gainsville/USA sowie den angeschlossenen ambulanten und chirurgischen Abteilungen.

$\mathrm{Zu}$ Beginn der zweimonatigen Beobachtung erhielten alle Mitglieder des medizinischen Teams eine 45-minütige Schulung über mögliche Fehlerquellen bei der Medikamentenverordnung. Dann wurden Berichte über mögliche Fehler bei der Arzneimittelverordnung sowohl durch das medizinische Personal als auch durch einen geschulten Mitarbeiter durch direktes Beobachten erfasst.

Im Rahmen der 589 erfassten Operationen wurden 20 Fehler bei der Arzneimittelverordnung registriert: zwei präoperative, vier operative, fünf während der Klinikeinweisung, zwei bei der Verlegung innerhalb verschiedener Abteilungen, vier bei der Klinikentlassung und drei postoperativ. Siebenmal wurde eine falsche Dosis gegeben, sechsmal die Medikamenteneinnahme ausgelassen, fünfmal das falsche Medikament verordnet, zweimal wurde die Applikationsseite falsch angegeben und in einem Fall ein überflüssiges Medikament verschrieben.

Häufigste Ursachen waren Unzulänglichkeiten im mündlichen und schriftlichen Informationsfluss, z.B. durch unsystematisches Erfassen der Grundmedikation des Patienten oder dem Erfassen von Allergien, das Übersehen von Patientenbesonderheiten wie z.B. Übergewicht, das bei einzelnen Medikamenten eine Dosisanpassung erfordert, sowie häufige Ablenkungen bzw. Unterbrechungen während der Konsultation. Auch die mangelnde Unterstützung des verordnenden
Personals z.B. durch elektronische Arzneimittelinformationen sowie die ungenügende Patienteninformation bei der Entlassung wurden moniert.

Fazit: Die Fehler bei der Arzneimittelverordnung im perioperativen HNO-Bereich unterscheiden sich in Art und Häufigkeit nicht von den allgemein beobachteten Verordnungsfehlern in der Chirurgie. In den meisten Fällen haben sie keine bleibenden negativen Konsequenzen für die Patienten. Verbessert werden sollte die Dokumentation der Grundmedikation und der neu verordneten Präparate sowie die Patienteninformation. Eine schnell erfassbare, elektronische Arzneimittelinformation kann zusätzlich helfen, Irrtümer bei der Wahl des Medikaments und der Dosis zu verhindern.

Rosenwasser R et al. Perioperative medication errors in otolaryngology. Laryngoscope 2010; 120: 1214-9

Kommentar: Fehler in der medikamentösen Behandlung während eines stationären Aufenthaltes zu einer HNO-Operation sind sicherlich ein heikles Thema, dennoch sollte nicht nur hinter vorgehaltener Hand darüber gesprochen werden. Alle beteiligten (Schwestern, HNO-Ärzte, Anästhesisten) waren in der vorliegenden Untersuchung aufgefordert, unter Anreiz von 10 Dollar pro Meldung, Fehler in der Medikation nicht anonymisiert zu melden. Festgestellt wurden 20 Medikationsfehler bei 589 chirurgischen Eingriffen.

Die Fehleranalyse ergab Unerfahrenheit, falsche Routine, falsche schriftliche Anweisungen, Rechenfehler, falsche Dosierung durch falsche Verdünnung, Nichtbeachten von Kontraindikation etc. Keiner der Fehler war schwerwiegend.

Studien wie diese sind selten und auf dem HNO-Gebiet noch seltener. Doch zeigen sie nun auf - wissenschaftlich belegt
Barbara Kreutzkamp
- was jeder Kliniker eigentlich weiß, dass Fehler in der täglichen Behandlungsroutine auftreten.

Studien dieser Art sind auch aus verschiedenen Sichtwinkeln angreifbar. So auch die Schlussfolgerung, die in eine bekannte Richtung geht: Mehr Kontrollen, bessere Dokumentation, mehr elektronische Entscheidungshilfen. Letztere wiederum - so wird negativ angemerkt - können jedoch auch dazu beitragen, dass andere Fehler entstehen: systematische Fehler, sorglose Auswahl, unnötige Medikation.

In dem Studien-Setting konnten natürlich schwere Fehler nicht entdeckt werden. Es war prädestiniert, nur kleine Fehler zu melden; vielleicht auch zu viele, denn jeder wusste ja, dass es Ziel war, Fehler zu finden. Und wieviele Medikationsfehler sind den Beobachtern entgangen? So wurden eigentlich nur die kleinen Fahrlässigkeiten erfasst.

Die klinische Erfahrung, Studien und vor allem medizinische Gerichtsprozesse zeigen, dass schwerwiegende Fehler jedoch häufig eine Kombination aus mehreren kleineren Fehlern sind, die in Reihe oder gehäuft zur gleichen Zeit auftreten. Daneben gibt es die "groben Fahrlässigkeiten", die schlichtweg nicht passieren dürfen. Verwechslung von links und rechts, die komplette Verwechslung eines Medikaments, eines Patientennamens oder das Verrechnen um eine Zehnerpotenz. An diesen schweren Fehlern müssen natürliche erste Maßnahmen angreifen.

Die von den Autoren diskutierten möglichen Verbesserungen, etwa eine elektronische Krankenakte, können auch solche groben Fehler nicht völlig ausschließen. Faktoren, wie Arbeitsüberlastung, Übermüdung, keine Zeit zur Übergabe, mangelnde Aufsicht und Personalmangel werden in der vorliegenden Arbeit nicht berücksichtigt. Eine Überregulierung durch noch mehr Papier, Formulare, Anweisungen und Richtlinien kann auch die Sicht auf die Dinge völlig verstellen. Daher muss eigentlich gelten: KIS! Keep it simple! Vertrauen in die richtige Anweisung ist gut, Kontrolle ist besser.

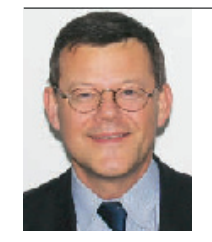

Prof Dr. med. Olaf Michel Universitair Ziekenhuis, Brüssel 